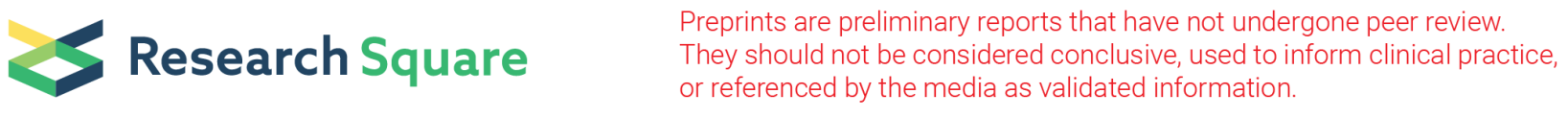

\title{
Phenotypic Sexual Dimorphism in Response to Dietary Fat Manipulation in C57BL/6J Mice
}

\author{
Isabel Casimiro \\ University of Chicago \\ Natalie D. Stull \\ Indiana Biosciences Research Institute \\ Sarah A. Tersey \\ University of Chicago \\ Raghavendra Mirmira ( $\square$ rmirmira@gmail.com ) \\ University of Chicago https://orcid.org/0000-0002-5013-6075
}

\section{Research}

Keywords: sexual dimorphism, obesity, high fat diet, western diet, C57/BL6J

Posted Date: June 18th, 2020

DOI: https://doi.org/10.21203/rs.3.rs-36103/v1

License: @ (1) This work is licensed under a Creative Commons Attribution 4.0 International License. Read Full License

Version of Record: A version of this preprint was published at Journal of Diabetes and its Complications on February 1st, 2021. See the published version at https://doi.org/10.1016/j.jdiacomp.2020.107795. 


\section{Abstract}

\section{Background:}

Obesity and the metabolic syndrome are increasingly prevalent in society and their complications and response to treatment exhibit sexual dimorphism. Mouse models of high fat diet-induced obesity are commonly used for both mechanistic and therapeutic studies of metabolic disease and diabetes. However, the inclusion of female mammals in obesity research has not been a common practice, and has resulted in a paucity of data regarding the effect of sex on metabolic parameters and its applicability to humans.

\section{Methods:}

Here we analyzed male and female C57BL/6J mice beginning at 4 weeks of age that were placed on a low-fat diet (LFD, $10 \%$ calories from fat), a Western Diet (WD, 45\% calories from fat), or a high fat diet (HFD, $60 \%$ calories from fat). Assessments of body composition, glucose homeostasis, insulin production, and energy metabolism, as well as histological analyses of pancreata were performed.

Results:

Both male and female C57BL/6J mice had similar increases in total percent body weight gain with both WD and HFD compared to LFD, however, male mice gained weight earlier upon HFD or WD feeding compared to female mice. Male mice exhibited a decrease in both food consumption and activity with either WD or HFD compared to LFD, whereas female mice did not exhibit any differences in food intake and minimal changes in locomotor activity on any diet. Glucose tolerance tests performed at 4, 12 and 20 weeks of dietary intervention revealed impaired glucose tolerance that was worse in male mice compared to females. Furthermore, male mice exhibited an increase in pancreatic $\beta$ cell area as well as reduced insulin sensitivity after HFD feeding compared to WD or LFD, whereas female mice did not.

\section{Conclusions:}

Male and female C57BL/6J mice exhibited strikingly different responses in weight, food consumption, locomotor activity, and $\beta$ cell adaptation upon dietary manipulation, with the latter exhibiting less striking phenotypic changes. We conclude that the nature of these responses emphasizes the need to contextualize studies of obesity pathophysiology and treatment with respect to sex.

\section{Background}

Metabolic syndrome is a cluster of medical conditions that include the presence of abdominal obesity, elevated blood pressure, hyperglycemia, and dyslipidemia (1). Obesity and metabolic syndrome are becoming increasingly prevalent worldwide, and can lead to numerous complications including, but not limited to cardiovascular disease, stroke, and type 2 diabetes (T2D). The causes of obesity and metabolic syndrome arise from a combination of both environmental factors and genetic factors. The most significant environmental factor that contributes to obesity and the subsequent development of metabolic syndrome is an increase in dietary fat intake, usually coupled with decreased physical activity. Indeed, metabolic syndrome has been called the "civilization syndrome" given its manifestations are associated with the urbanized Western lifestyle (2).

Diets rich in fat induce obesity in humans and in mice (3). Thus, while several animal models of obesity and metabolic syndrome exist (4-6), one of the most commonly used murine models involves the use of diets high in fat (30-78\% of total energy intake from fat) to induce obesity and its related comorbidities (7-9). Diet induced obesity (DIO) models are highly valuable models, as they can be used to study adipose tissue development and insulin resistance in genetically manipulated mice or alongside therapeutic drugs. Whereas different types of high fat diets are used in the metabolic literature, the two most commonly used include a $42-45 \%$ calorie from fat referred to as the "Western Diet (WD)", and the "High Fat Diet (HFD)," which is comprised of $60 \%$ calories from fat. However, direct metabolic comparisons between the WD and HFD in males and female mouse models of obesity are lacking.

Mouse strain differences affect the response to diet manipulation (10). The C57BL/6J strain is perhaps the most common inbred strain used in metabolic experiments, owing to its high susceptibility to DIO and insulin resistance $(11,12)$. The C57BL/6J strain is viewed as a good model to mimic metabolic syndrome in humans because mice of this strain develop obesity, hyperinsulinemia, hyperglycemia and hypertension when allowed ad libitum access to a diet with high fat content (13). Importantly, whereas sexual dimorphism is known to exist in mouse models of obesity $(14,15)$, few studies have directly examined the metabolic effects of DIO in female mice. Several epidemiological studies in humans have shown sex differences in obesity, cardiometabolic risk factors, response to medications, and development of diabetic complications (16-18). Whereas it has been widely reported that cardiovascular risk is higher in men, in the 
setting of diabetes this sex difference completely reverses with a $44 \%$ higher relative risk of coronary heart disease in women (19). Thus, increased understanding of the biological basis of sexual dimorphisms in metabolic disease is imperative.

In this study, we examined the metabolic differences in both male and female C57BL/6J mice fed commonly used diets in DIO studies. We show that males and females fed a WD and a HFD exhibit significant differences in weight gain, glucose tolerance, and insulin resistance over a 22-week course of feeding in comparison to a control low fat diet (LFD, 10\% calories from fat). Furthermore, we also show that major sex differences exist among caloric intake, physical activity, respiratory exchange ratio, and pancreatic morphology between male and female mice. Overall, our data suggest that for studies using DIO models, the choice of diet and the inclusion of both sexes are crucial parameters for the potential applicability to human disease.

\section{Methods}

Animals

Male and female C57BL/6J mice were purchased from Jackson Laboratories. Mice were maintained at the Indiana University vivarium according to protocols approved by the Institutional Animal Care and Use Committee. At 4 weeks of age, animals were fed either a low-fat diet containing 10\% calories from fat (LFD; cat\# D12450J, Research Diets, New Brunswick, NJ USA), a Western diet containing 45\% calories from fat (WD; cat\# D12451, Research Diets, New Brunswick, NJ, USA), or a high fat diet containing 60\% calories from fat (HFD; cat\# D12492, Research Diets, New Brunswick, NJ, USA). All mice were kept on a standard 12 h:12 h light-dark cycle with ad libitum access to chow and water.

Metabolic Testing

Body composition was analyzed by quantitative magnetic resonance imaging using EchoMRI Body Composition Analyzer (EchoMRI, LLC, Houston, Texas). Indirect calorimetry measures were performed using a TSE systems PhenoMaster Metabolism Research Platform (Chesterfield, MO) equipped with calorimetry, feeding/drinking, and activity monitoring as described (20). Glucose tolerance tests (GTTs) were performed following a 16 hour fast and intraperitoneal injection of $2 \mathrm{~g} / \mathrm{kg}$ lean mass of D-glucose solution. Blood was sampled from the tail vein at 0, 10, 20, 30, 60, 90, and 120 min, and glucose was measured using a glucometer (AlphaTRAK, Abbott Park, IL, USA). Insulin tolerance tests (ITTs) were performed on 2 hour fasted mice following an intraperitoneal injection of $0.75 \mathrm{U} / \mathrm{kg}$ lean mass Humulin-R insulin (Lilly, Indianapolis, IN, USA). Blood glucose was measured from tail vein blood at 0, 15, 30, 45, and 60 min.

Immunohistochemistry and immunofluorescence

Mice were euthanized and serum/pancreata were harvested as previously described (21). Immunohistochemical analysis of insulin (Santa Cruz Biotechnology) was performed as previously described (22). $\beta$-cell area was calculated by quantifying insulin positive area and dividing by pancreas cell positive area using CV-H1X software (Keyence, Mechelen, Belgium).

\section{Statistical analysis}

All data are presented as the mean \pm SEM. Two-tailed t tests were used to compare groups where two means were compared and one-way ANOVA (with Tukey's post-test) was used for comparisons involving more than two conditions. Prism 8 software (GraphPad) was used for all statistical analyses. Differences were considered statistically significant at $p<0.05$.

\section{Results}

\section{Sexual dimorphism in the rate of weight gain upon dietary manipulation.}

To assess sex differences in response to DIO, we challenged both male and female C57BL/6J mice with three different diets that are commonly used in DIO studies for a total of 22 weeks: 1) 10\% calories from fat (low fat diet, LFD), 2) $45 \%$ calories from fat (Western Diet, WD), and 3) $60 \%$ calories from fat (high fat diet, HFD). The LFD was sucrose matched to the HFD and the primary source of fat was lard for each diet (Table 1). To determine changes in body composition over time, mice were weighed weekly and body composition was measured by quantitative magnetic resonance imaging at 4, 12 and 20 weeks of dietary intervention. As expected, both male and female mice gained significantly more body weight when fed a WD or HFD compared to LFD-fed mice. However, male mice gained significantly more body weight after only 2 weeks of HFD feeding compared to mice fed a LFD ( $21.98 \mathrm{~g} \pm 0.444$ vs $19.04 \pm 0.306$ respectively, $\mathrm{p}=0.0001$ ), whereas male mice fed a WD showed a significant increase in body weight after 6 weeks of WD feeding compared to mice fed a LFD $(27.59 \mathrm{~g} \pm 0.825$ vs $24.24 \pm 0.466$, respectively, $p=0.0033)$ (Fig 1A). Female mice, by contrast, did not show a significant weight 
increase until 11 weeks of HFD feeding compared to LFD-fed mice $(25.79 \mathrm{~g} \pm 1.175$ vs $21.20 \pm 0.346$ respectively, $p=0.0038)$, and 13 weeks of WD feeding compared to LFD fed mice $(24.40 \mathrm{~g} \pm 0.829$ vs $20.83 \pm 0.375$, respectively $p=0.0017)$ (Fig $1 \mathrm{C})$. Similar to other published data (23), female mice did not gain as much weight as male mice early during feeding of either WD or HFD. However, at the end of the 22-week challenge, both male and female mice gained the same proportion of weight ( $252 \%$ increase in total body weight in males compared to $236 \%$ increase in females when fed a WD, $p=0.899, n=6-8$, and $267 \%$ increase in body weight in males compared to $228 \%$ body weight increase in females fed a HFD, $p=0.166, n=6-8$ (Fig. 1 B, D). Fat mass was significantly increased in male mice fed either WD or HFD at 12 and 20 weeks of feeding (Fig 1E), while lean mass did not significantly change in males until 20 weeks of HFD feeding compared to LFD-fed controls and WD fed mice (Fig 1F). Female mice showed increased fat mass only at 20 weeks with either HFD and WD feeding (Fig 1G), and exhibited significantly more lean mass at 20 weeks on either a HFD or WD compared to LFD-fed mice (Fig 1H).

\section{Sexual dimorphism in food intake and activity upon dietary manipulation.}

Next, mice were placed in metabolic cages after 8 and again after 16 weeks of diet to monitor food intake, activity levels, and respiratory exchange. Measurements were obtained over a continuous 48 hour period at 8 and 16 weeks of diet. Dietary intervention with WD or HFD in both male and female mice led to sexual dimorphism in the quantity of food consumed during light and dark cycles. In particular, male mice fed either a WD or HFD had reduced food consumption during both light and dark cycles compared to LFD-fed controls at 8 weeks (Fig 2A). In terms of locomotor activity, male mice exhibited reduced activity levels after 8 weeks of WD or HFD compared to LFD-fed controls (Fig 2B, C). Female mice did not exhibit any differences in total grams of food consumption on either diet at 8 weeks (Fig 2D), and they reduced their activity levels upon WD feeding during the light cycle at 8 weeks, but not during the dark (active) cycle with WD or HFD feeding (Fig. 2E, F). At 16 weeks of dietary intervention, male mice on WD or HFD continued to eat significantly less than LFD-fed mice during the light cycle and significantly less on the HFD during the dark cycle (Fig 2G). At 16 weeks of DIO, locomotor activity levels in male mice was also reduced during the light cycle in mice fed both diets, and in mice fed the WD during the dark cycle (Fig $2 \mathrm{H}$, I). Female mice, by striking contrast, did not change their food intake or activity levels during the light or dark cycles at 16 weeks of diet (Fig 2J-L). These results are in agreement with the body of literature demonstrating that consumption of a HFD results in reduced locomotor activity in C57/BL6J male mice compared to female mice $(23,24)$,

The respiratory exchange ratio (RER) measures inherent composition and utilization of fats, carbohydrates, and proteins as they are converted to energy substrate. An RER of 1 indicates that only carbohydrates are being used as substrate, and an RER of 0.7 indicates that only fat is being used as substrate (25). Consistent with the utilization of fat as substrate, male mice fed WD or HFD exhibited significantly reduced RER (compared to LFD) in both light and dark cycles at 8 weeks of diet (Fig. 3A, C). Female mice also showed a significantly reduced RER in the dark cycle at 8 weeks of diet and showed no statistical difference in RER when fed a WD during the light cycle (Fig 3B, D). At 16 weeks of diet male mice continued to show reduced RER in both light and dark cycles under both WD and HFD feeding compared to LFD-fed mice (Fig 3E, G). Similarly, to 8 weeks of diet, females showed a reduction of RER in the dark cycle when fed a WD or HFD at 16 weeks and reduced RER when fed a WD only during the light cycle (Fig 3F, H). Collectively, these data demonstrate that consumption of diets high in dietary fat leads to changes in locomotor activity, food intake, and a change in the animal's primary fuel source. Furthermore, while mice of either sex fed a LFD demonstrated a clear peak of nocturnal increased food intake with concomitant peaks in RER during the nocturnal cycle, HFD and WD feeding attenuated the diurnal peak pattern of feeding behavior (Fig 3A-B, E-F). This is consistent with reports that diets high in fat disrupt circadian rhythmicity in mice $(26,27)$.

\section{Effect of DIO on glucose tolerance and insulin resistance in male and female mice.}

To assess changes in glucose homeostasis, glucose tolerance tests (GTTs) were performed at 4, 12, and 20 weeks of dietary intervention. After 4 weeks of diet, male mice on either a WD and HFD had worsening glucose tolerance compared to LFD-fed control mice (Fig. 4A, B), an observation that persisted at weeks 12 and 20 (Fig. 4E-F, I-J). Female mice also showed worsening glucose tolerance on either the WD or the HFD at 4 and 12 weeks of feeding (Fig. 4C-D, G-H). However, at 20 weeks of dietary intervention while female mice had worsening glucose tolerance on the HFD, the area under the curve (AUC) analysis was no different in WD-fed compared to LFD-fed female mice (Fig $4 \mathrm{~K}, \mathrm{~L}$ ). Furthermore, it is worth noting that area under the curve (AUC) values calculated from female GTTs on a WD or HFD intervention were lower compared to male AUC values on either the WD or HFD at all time points, suggesting an inherent resistance of females to glycemic deterioration caused by dietary intervention.

To determine if differences in insulin resistance contributed to the differences observed in glucose tolerance, insulin tolerance tests (ITTs) were performed at the end of dietary intervention at 22 weeks. Final ITTs performed on male mice fed either the WD or HFD showed reduced insulin sensitivity compared to LFD-fed mice (Fig 5A, B). Whereas insulin sensitivity was not as markedly affected. ITTs revealed that female mice on WD and HFD exhibited minimal to no impairment in insulin tolerance compared to females on LFD (Fig 5C, D).

Page $4 / 13$ 


\section{Sexual dimorphism in adaptive $\beta$-cell hyperplasia}

Increases in insulin resistance result in increases in islet $\beta$-cell mass/area as an adaptive mechanism to meet peripheral insulin demand (28). To assess for potential changes in islet morphometry, pancreata were removed after 22 weeks of dietary intervention and immunostained for insulin. Although male mice fed a WD did not show statistically significant changes in $\beta$-cell area compared to LFDfed mice, male mice fed a HFD had significantly increased $\beta$-cell area (Fig. 6, top panel). Notably, HFD-fed female mice trended towards an increase in $\beta$-cell area $(p=0.0706)$, but neither WD nor HFD resulted in significantly altered $\beta$-cell mass in females (Fig. 6 , bottom panel), a finding consistent with the lack of changes in insulin resistance.

\section{Discussion}

The epidemic rise in obesity worldwide is a major public health concern in both men and women. Mouse models used for the study of obesity have been limited by the underrepresentation of female animals despite well-established sex differences in human disease and treatment outcomes (29-31). The goal of this study was to interrogate how male and female C57BL/6J mice responded to DIO when challenged with commonly used diets in metabolic research. The data presented here showed that sexual dimorphisms exist between C57BL/ 6 mice in terms of (i) the rate of weight gain, (ii) metabolic parameters, and (iii) insulin sensitivity with corresponding pancreatic $\beta$ cell adaptation. Several prior studies have closely examined weight gain following high fat diet feeding of male mice, and have observed rapid weight gain within 1-2 weeks of initiating diet $(20,32)$. Our studies presented here in male mice are consistent with these prior studies, with male mice demonstrating rapid weight gain (within 2 weeks on HFD and 6 weeks on WD). Notably, female mice appeared more resistant to fat accumulation and the T2D-promoting effects of the WD or HFD during the early weeks on these diets. Published reports have documented the increased susceptibility of male mice to obesity compared to females (33). In a study by Gelineau et al., the authors investigated behavioral and physiological responses to consumption of chow diet (13\% fat), a low-fat diet (10\% fat) and a high fat diet (60\% fat) in the presence of water or $10 \%$ alcohol in C57BL/6J mice. Interestingly, this study demonstrated earlier weight gain with reduced activity in males, as well as better glucose tolerance in females in response to a high fat diet (23). However, direct comparisons including insulin sensitivity between male and female C57/BL6 mice on a low fat diet, WD or HFD have not been previously adequately evaluated. Although our study shows that two of the commonly used metabolic diets in the obesity literature have different effects in male and female C57BL/6J mice, both diets result in worsening metabolic parameters in males. Studies that have documented the increased propensity of male mice to develop obesity have shown this effect might be due to hormonal physiological differences, as ovariectomy can eliminate the protection against weight gain in female mice (15). In humans, studies have shown that post-menopausal women are more susceptible to develop obesity and T2D compared to pre-menopausal women (34). However, while sexual dimorphism has been mainly attributed to gonadal hormones, chromosomal genetic differences likely also underlie physiological sex differences $(35,36)$.

The rate of weight gain differences between male and female mice was not easily explained by the amount of food consumption alone. Our results show that male C57BL/6J mice reduce their food consumption upon feeding with either a WD or HFD, while female mice had the same food consumption compared to LFD-fed mice at 8 and 16 weeks of feeding. A striking difference was noted in activity levels, as well. Male mice reduced activity on WD and HFD, whereas female mice largely maintained the same level of activity on these diets as compared to LFD. RER analysis revealed that both male and female mice switched their fuel utilization source towards fatty acids upon feeding with either WD or HFD. However, diurnal RER peak patterns were attenuated in both males and females at 8 and 16 weeks of dietary challenge. These results suggest inherent differences in feeding and activity behavior between male and female mice, such that male mice are more vulnerable to weight gain and fat mass accumulation during DIO compared to females.

Another striking finding in our study was the apparent lack of change in $\beta$-cell area \% in female mice following WD and HFD. In the setting of insulin resistance, the pancreatic islets respond by increasing insulin production and release, in part through compensatory $\beta$-cell mass expansion $(37,38)$. Our findings in male mice show clear increases in $\beta$-cell area \% upon HFD feeding (and a trend toward an increase upon WD feeding), a finding consistent with the insulin resistance observed in these animals in ITTs. Nevertheless, this compensation was insufficient to normalize glucose homeostasis in these animals and is a finding in agreement with prior studies that suggest inherent defects in $\beta$-cell function in male C57BL/6J mice (20). Notably, in our study, ITTs revealed that female mice were protected from the development of insulin resistance upon WD or HFD compared to male mice. The lower insulin resistance in females upon WD and HFD likely explains their preserved $\beta$-cell area \%. Nevertheless, female mice on WD or HFD did experience mild glucose intolerance, suggesting that defects in $\beta$-cell function related to oxidative stress and suppression of mRNA translation, as we have described previously, may be occurring in female mice (39). 
There are some limitations to this study that should be noted. First, C57BL/6J mice were used. There are many other mouse strains, including C57BL/6NJ mice, that likely will respond to HFD feeding differently. The C57BL/6J mice (but not C57BL/6NJ mice) express a loss-of-function mutation in the gene encoding nicotinamide nucleotide transhydrogenase (Nnt), which is associated with a reduction in glucose-stimulated insulin secretion from $\beta$-cells. Prior studies comparing these strains have shown that C57BL/6J male mice gain more weight and have worsening glucose tolerance at 14 weeks of HFD feeding compared to male $6 \mathrm{NJ}$ mice (40). In a study that included female mice, C57BL/6NJ female mice were found to have lower basal weight and blood glucose levels compared to males, but gained weight and glucose intolerance at a rate similar to male mice (41). The C57BLKS/J (BLKS) strain is a strain of mice that arose from the C57BL/6J background and has been characterized to be more resistant to DIO. A comparison of C57BL/6J and BLKS male mice showed that BLKS mice gain less weight and adiposity in response to a HFD by restricting food intake and increasing activity, which ultimately protected against HFD-induced glucose intolerance (20). Whether female BLKS mice exhibit improved metabolic parameters compared to their male counterparts has not been investigated.

\section{Conclusions}

Despite recent calls by the National Institutes of Health for the inclusion of females in research studies, a recent report showed that although there has been an increase in the inclusion of females across major disciplines in the last 10 years, most studies have omitted sex-based or single sex analyses (42). Biomedical research has historically neglected the impact of sex in biological processes. Our study provides direct evidence that sexual dimorphisms exist in mouse models of DIO, and that further interrogation of these dimorphisms might lead to better insight into human obesity.

\section{Abbreviations}

GTT: glucose tolerance test

ITT: insulin tolerance test

T2D: Type 2 diabetes

HFD: High fat diet

WD: Western diet

LFD: Low fat diet

DIO: Diet induced obesity

RER: Respiratory Exchange Ratio

Nnt: nicotinamide nucleotide transhydrogenase

AUC: Area under the curve

\section{Declarations}

\section{Ethics approval and consent to participate:}

Not applicable, since no humans were studied. Animal ethical approval was obtained from the Indiana University Institutional Animal Care and Use Committee.

Consent for publication:

Not applicable

Availability of data and materials:

The datasets used and/or analyzed during the current study are available from the corresponding author on reasonable request.

Competing interests:

Page 6/13 
The authors declare that they have no competing interests.

\section{Funding:}

This work was supported by National Institutes of Health grants R01 DK060581, R01 DK124906 and by the Indiana Biosciences Research Institute (to RGM). This study utilized core resources provided by National Institutes of Health grants P30 DK097512 (to Indiana University), P30 DK020595 (to University of Chicago) and T32DK007011 (to IC).

\section{Acknowledgements:}

We thank Jen Nelson for technical assistance with the beta cell immunostaining experiments.

\section{Authors' contributions:}

NDS and SAT performed the experiments. IC, NDS, SAT, and RGM analyzed and interpreted the data. IC, SAT, and RGM wrote the manuscript. All authors read and approved the final manuscript.

\section{References}

1. Kassi E, Pervanidou P, Kaltsas G, Chrousos G. Metabolic syndrome: definitions and controversies. BMC Med. 2011 May 5;9:48.

2. Björntorp P. Abdominal Fat Distribution and the Metabolic Syndrome. J Cardiovasc Pharmacol. 1992;20:S26.

3. Bourgeois F, Alexiu A, Lemonnter D. Dietary-induced obesity: effect of dietary fats on adipose tissue cellularity in mice. Br J Nutr. 1983 Jan;49(1):17-26.

4. Lutz TA, Woods SC. Overview of Animal Models of Obesity. Curr Protoc Pharmacol. 2012;58(1):5.61.1-5.61.18.

5. Heydemann A. An Overview of Murine High Fat Diet as a Model for Type 2 Diabetes Mellitus. J Diabetes Res. 2016;2016:1-14.

6. Kleinert M, Clemmensen C, Hofmann SM, Moore MC, Renner S, Woods SC, et al. Animal models of obesity and diabetes mellitus. Nat Rev Endocrinol. 2018 Mar;14(3):140-62.

7. Scroyen I, Hemmeryckx B, Lijnen HR. From mice to men - mouse models in obesity research: What can we learn? Thromb Haemost. 2013;110(10):634-40.

8. Buettner R, Schölmerich J, Bollheimer LC. High-fat Diets: Modeling the Metabolic Disorders of Human Obesity in Rodents. Obesity. 2007;15(4):798-808.

9. Hariri N, Thibault L. High-fat diet-induced obesity in animal models. Nutr Res Rev. 2010 Dec;23(2):270-99.

10. Kanasaki K, Koya D. Biology of Obesity: Lessons from Animal Models of Obesity. J Biomed Biotechnol [Internet]. 2011 [cited 2020 Jun 4];2011. Available from: https://www.ncbi.nlm.nih.gov/pmc/articles/PMC3022217/

11. Surwit RS, Kuhn CM, Cochrane C, McCubbin JA, Feinglos MN. Diet-Induced Type II Diabetes in C57BL/6J Mice. Diabetes. 1988 Sep 1;37(9):1163-7.

12. Collins S, Martin TL, Surwit RS, Robidoux J. Genetic vulnerability to diet-induced obesity in the C57BL/6J mouse: physiological and molecular characteristics. Physiol Behav. 2004 Apr 1;81(2):243-8.

13. Rebuffé-Scrive M, Surwit R, Feinglos M, Kuhn C, Rodin J. Regional fat distribution and metabolism in a new mouse model (C57BL/6J) of non-insulin-dependent diabetes mellitus. Metabolism. 1993 Nov;42(11):1405-9.

14. Yang Y, Smith DL, Keating KD, Allison DB, Nagy TR. Variations in body weight, food intake and body composition after long-term highfat diet feeding in C57BL/6J mice. Obesity. 2014;22(10):2147-55.

15. Hong J, Stubbins RE, Smith RR, Harvey AE, Núñez NP. Differential susceptibility to obesity between male, female and ovariectomized female mice. Nutr J. 2009 Feb 17;8(1):11.

16. Gerdts E, Regitz-Zagrosek V. Sex differences in cardiometabolic disorders. Nat Med. 2019 Nov;25(11):1657-66.

17. Seghieri G, Policardo L, Anichini R, Franconi F, Campesi I, Cherchi S, et al. The Effect of Sex and Gender on Diabetic Complications. Curr Diabetes Rev. 2017;13(2):148-60.

18. Wilding JPH, Overgaard RV, Jacobsen LV, Jensen CB, le Roux CW. Exposure-response analyses of liraglutide 3.0 mg for weight management. Diabetes Obes Metab. 2016 May 1;18(5):491-9.

19. Lyon A, Jackson EA, Kalyani RR, Vaidya D, Kim C. Sex-specific differential in risk of diabetes-related macrovascular outcomes. Curr Diab Rep. 2015 Nov;15(11):85. 
20. Sims EK, Hatanaka M, Morris DL, Tersey SA, Kono T, Chaudry ZZ, et al. Divergent compensatory responses to high-fat diet between C57BL6/J and C57BLKS/J inbred mouse strains. Am J Physiol-Endocrinol Metab. 2013 Oct 29;305(12):E1495-511.

21. Evans-Molina C, Robbins RD, Kono T, Tersey SA, Vestermark GL, Nunemaker CS, et al. Peroxisome Proliferator-Activated Receptor Y Activation Restores Islet Function in Diabetic Mice through Reduction of Endoplasmic Reticulum Stress and Maintenance of Euchromatin Structure. Mol Cell Biol. 2009 Apr 15;29(8):2053-67.

22. Francis J, Chakrabarti SK, Garmey JC, Mirmira RG. Pdx-1 Links Histone H3-Lys-4 Methylation to RNA Polymerase II Elongation during Activation of Insulin Transcription. J Biol Chem. 2005 Oct 28;280(43):36244-53.

23. Gelineau RR, Arruda NL, Hicks JA, Pina IMD, Hatzidis A, Seggio JA. The behavioral and physiological effects of high-fat diet and alcohol consumption: Sex differences in C57BL6/J mice. Brain Behav. 2017;7(6):e00708.

24. Krishna S, Keralapurath MM, Lin Z, Wagner JJ, de La Serre CB, Harn DA, et al. Neurochemical and electrophysiological deficits in the ventral hippocampus and selective behavioral alterations caused by high-fat diet in female C57BL/6 mice. Neuroscience. 2015 Jun 25;297:170-81.

25. Engelking L R. Textbook of Veterinary Physiological Chemistry [Internet]. Vol. Third. Elsevier; 2015 [cited 2020 Jun 4]. 786 p. Available from: https://reader.elsevier.com/reader/sd/pii/B9780123919090500785?

token=B206A4D164715FBD1F0C0A972AFDB0F7F09570200E08D752A9BCA594B9E95480FD2967A78F81399FB567085FE16DBF43

26. Kohsaka A, Laposky AD, Ramsey KM, Estrada C, Joshu C, Kobayashi Y, et al. High-Fat Diet Disrupts Behavioral and Molecular Circadian Rhythms in Mice. Cell Metab. 2007 Nov 7;6(5):414-21.

27. Engin A. Circadian Rhythms in Diet-Induced Obesity. In: Engin AB, Engin A, editors. Obesity and Lipotoxicity [Internet]. Cham: Springer International Publishing; 2017 [cited 2020 Jun 11]. p. 19-52. (Advances in Experimental Medicine and Biology). Available from: https://doi.org/10.1007/978-3-319-48382-5_2

28. Tersey SA, Maier B, Nishiki Y, Maganti AV, Nadler JL, Mirmira RG. 12-lipoxygenase promotes obesity-induced oxidative stress in pancreatic islets. Mol Cell Biol. 2014 Oct 1;34(19):3735-45.

29. Putting gender on the agenda. Nature. 2010 Jun;465(7299):665-665.

30. Heinrich J. Most drugs withdrawn in recent years had greater health risks for women. Gen Account Off-01-286R 2001; 2001.

31. Clayton JA, Collins FS. Policy: NIH to balance sex in cell and animal studies. Nat News. 2014 May 15;509(7500):282.

32. Peyot M-L, Pepin E, Lamontagne J, Latour MG, Zarrouki B, Lussier R, et al. Beta-cell failure in diet-induced obese mice stratified according to body weight gain: secretory dysfunction and altered islet lipid metabolism without steatosis or reduced beta-cell mass. Diabetes. 2010 Sep;59(9):2178-87.

33. Grove KL, Fried SK, Greenberg AS, Xiao XQ, Clegg DJ. A microarray analysis of sexual dimorphism of adipose tissues in high-fat-dietinduced obese mice. Int J Obes. 2010 Jun;34(6):989-1000.

34. Shi H, Clegg DJ. Sex differences in the regulation of body weight. Physiol Behav. 2009 May 25;97(2):199-204.

35. Zore T, Palafox M, Reue K. Sex differences in obesity, lipid metabolism, and inflammation-A role for the sex chromosomes? Mol Metab. 2018;15:35-44.

36. Link JC, Reue K. Genetic Basis for Sex Differences in Obesity and Lipid Metabolism. Annu Rev Nutr. 2017 21;37:225-45.

37. Steil GM, Trivedi N, Jonas JC, Hasenkamp WM, Sharma A, Bonner-Weir S, et al. Adaptation of beta-cell mass to substrate oversupply: enhanced function with normal gene expression. Am J Physiol Endocrinol Metab. 2001 May;280(5):E788-796.

38. Prentki M, Nolan CJ. Islet $\beta$ cell failure in type 2 diabetes. J Clin Invest. 2006 Jul 3;116(7):1802-12.

39. Hatanaka M, Anderson-Baucum E, Lakhter A, Kono T, Maier B, Tersey SA, et al. Chronic high fat feeding restricts islet mRNA translation initiation independently of ER stress via DNA damage and p53 activation. Sci Rep. 2017 Jun 19;7(1):3758.

40. Nicholson A, Reifsnyder PC, Malcolm RD, Lucas CA, MacGregor GR, Zhang W, et al. Diet-induced obesity in two C57BL/6 substrains with intact or mutant nicotinamide nucleotide transhydrogenase (Nnt) gene. Obes Silver Spring Md. 2010 Oct;18(10):1902-5.

41. Ingvorsen C, Karp NA, Lelliott CJ. The role of sex and body weight on the metabolic effects of high-fat diet in C57BL/6N mice. Nutr Diabetes. 2017 Apr;7(4):e261-e261.

42. Woitowich NC, Beery A, Woodruff T. A 10-year follow-up study of sex inclusion in the biological sciences. eLife [Internet]. [cited 2020 Jun 11];9. Available from: https://www.ncbi.nlm.nih.gov/pmc/articles/PMC7282816/

\section{Tables}




\begin{tabular}{|l|c|c|c|}
\hline & $\begin{array}{c}\text { LFD } \\
\text { D12450J }\end{array}$ & $\begin{array}{c}\text { WD } \\
\text { D12451 }\end{array}$ & $\begin{array}{c}\text { HFD } \\
\text { D12492 }\end{array}$ \\
\hline Component & gm\% (kcal \%) & gm\% (kcal \%) & gm\% (kcal \%) \\
\hline Protein (gm\%) & $19.2(20)$ & $24(20)$ & $26.2(20)$ \\
\hline $\begin{array}{l}\text { Carbohydrate } \\
(\text { gm\%) }\end{array}$ & $67.3(70)$ & $41(35)$ & $26.3(20)$ \\
\hline Fat (gm\%) & $4.3(10)$ & $24(45)$ & $34.9(60)$ \\
\hline Ingredient & $\mathrm{g} \mathrm{(kcal)}$ & $\mathrm{g} \mathrm{(kcal)}$ & $\mathrm{g} \mathrm{(kcal)}$ \\
\hline Corn Starch & $506.2(2024.8)$ & $72.8(291)$ & $0(0)$ \\
\hline Maltodextrin 10 & $125(500)$ & $100(400)$ & $125(500)$ \\
\hline Sucrose & $68.8(275.2)$ & $172.8(691)$ & $68.8(275.2)$ \\
\hline Soybean Oil & $25(225)$ & $25(225)$ & $25(225)$ \\
\hline Lard & $20(180)$ & $177.5(1598)$ & $245(2205)$ \\
\hline
\end{tabular}

\section{Table 1: Composition of Diets}

Figures

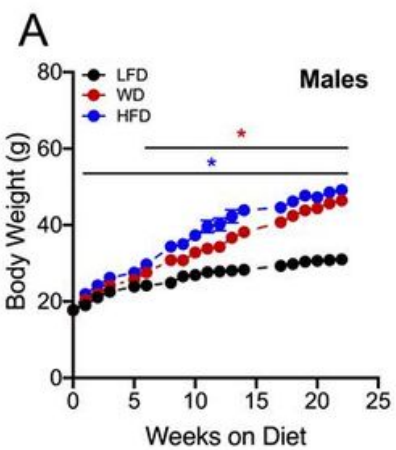

B

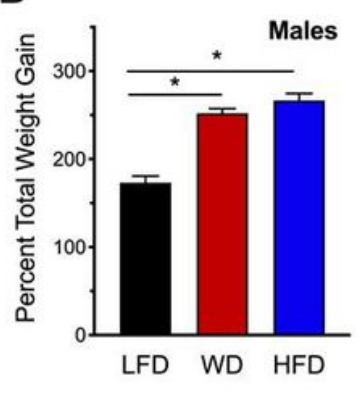

F

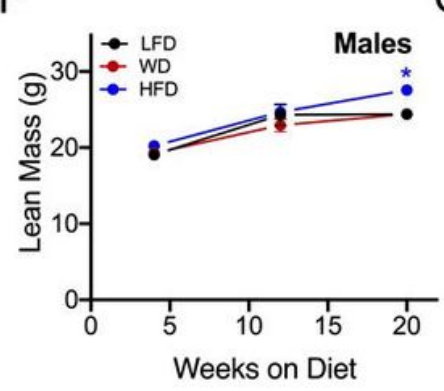

C
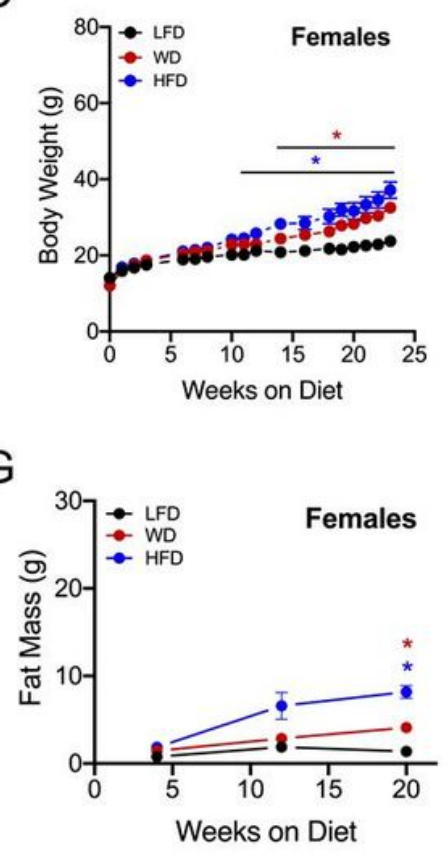

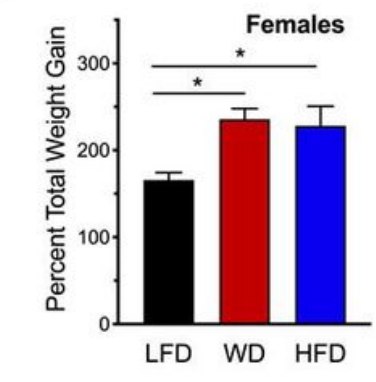

$\mathrm{H}$

D

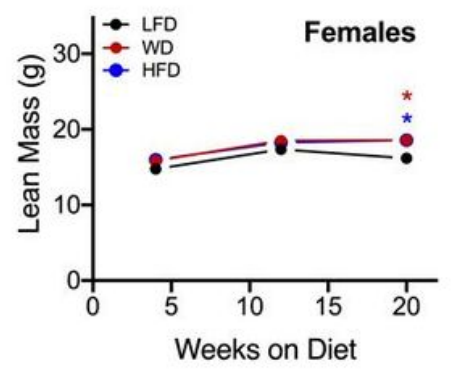

Figure 1

High fat dietary challenge results in faster weight gain in male mice compared to females. Male and female C57BL6/J mice were placed on an intervention diet starting at 4 weeks of age. (A) Weekly body weights of male mice; (B) Percent total weight gain in males at 22 weeks of dietary intervention; (C) Weekly body weights of female mice; (D) Percent total weight gain in females at 22 weeks of dietary intervention; (E) Male fat mass at 4, 12 and 20 weeks of dietary intervention; (F) Lean mass at 4, 12, and 20 weeks of dietary intervention in male mice; (G) Female fat mass at 4, 12 and 20 weeks of dietary intervention; $(H)$ Female lean mass at 4, 12, and 20 weeks of dietary 
intervention; Results are displayed as means \pm SEM; means were compared by one-way ANOVA followed by Tukey's posttest; *, $p<0.05$, $\mathrm{n}=4-8$.

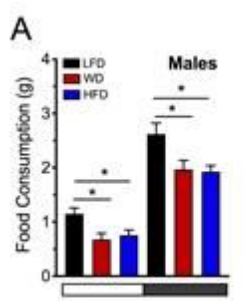

D
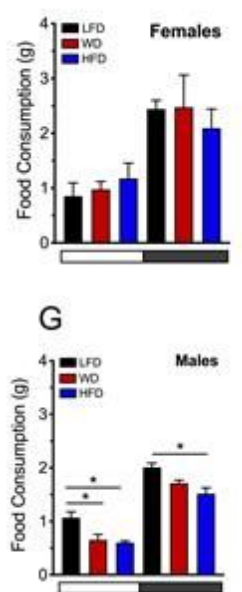

$\mathrm{J}$

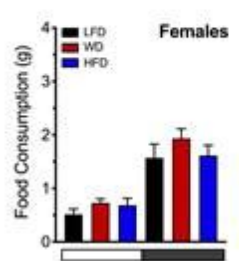

B

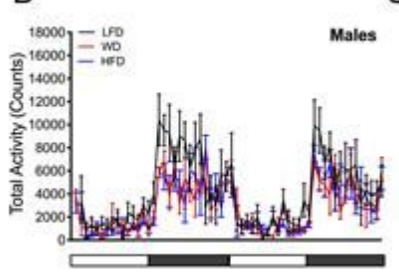

$E$

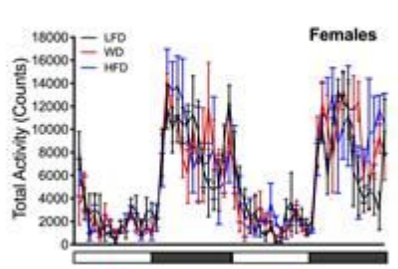

$\mathrm{H}$

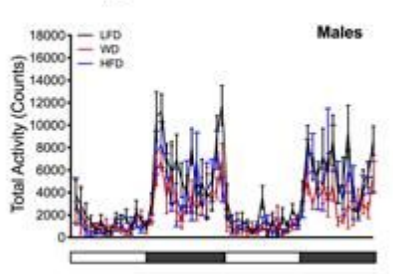

$\mathrm{K}$

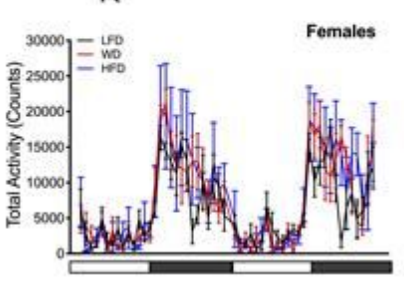

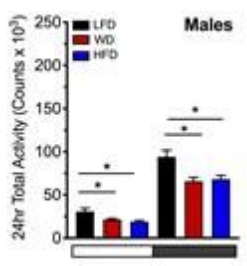

$\mathrm{F}$

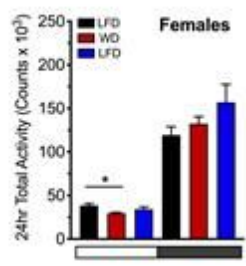

।

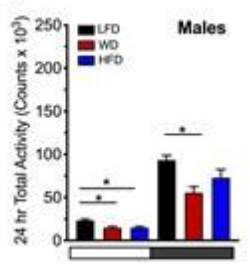

L

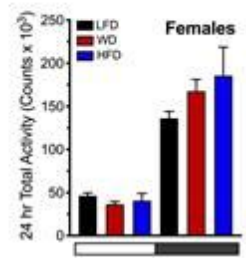

Figure 2

Food intake and activity levels are decreased in males challenged with diet induced obesity. Male and female C57BL6/J mice were placed on a dietary intervention starting at 4 weeks of age. Food intake was monitored and activity was assessed by recording the number of beam breaks occurring in the $x+y$ - and $z$-axes at 8 and 16 weeks of diet. (A) Food intake of male mice at 8 weeks of dietary intervention; (B) Male locomotor activity during the light and dark cycles at 8 weeks of dietary intervention (48 hours depicted); (C). Male locomotor activity at 8 weeks of dietary intervention (total beam break counts in 24 hours); (D) Food intake of female mice at 8 weeks of dietary intervention; (E) Female locomotor activity during the light and dark cycles at 8 weeks of dietary intervention (48 hours depicted); (F). Female locomotor activity at 8 weeks of dietary intervention (total beam break counts in 24 hours); (G) Food intake of male mice at 16 weeks of dietary intervention; $(\mathrm{H})$ Male locomotor activity during the light and dark cycles at 16 weeks of dietary intervention; (I) Male locomotor activity at 16 weeks of dietary; $(\mathrm{J})$ Food intake of female mice at 16 weeks of dietary intervention; (K) Female locomotor activity during the light and dark cycles at 16 weeks of dietary intervention; (L) Female locomotor activity at 16 weeks of dietary intervention. Horizontal white bars denote light cycles $(0700-1900)$ and dark gray horizontal bars denote dark cycles $(1900-0700)$. Results are displayed as means \pm SEM; Means were compared by one-way ANOVA followed by Tukey's posttest; *, $p<0.05, n=9-10$. 
A

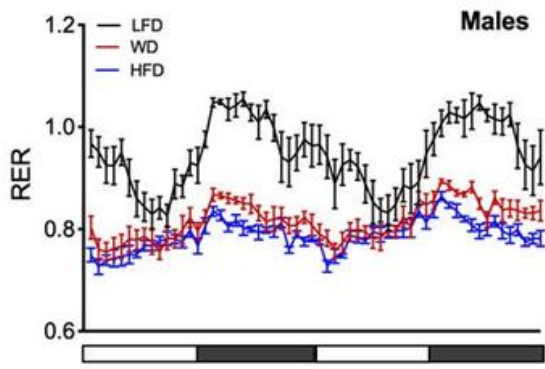

E

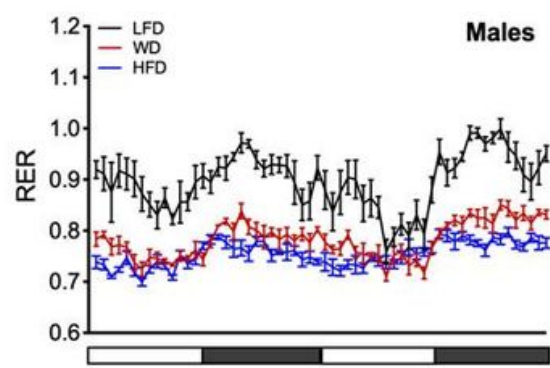

B

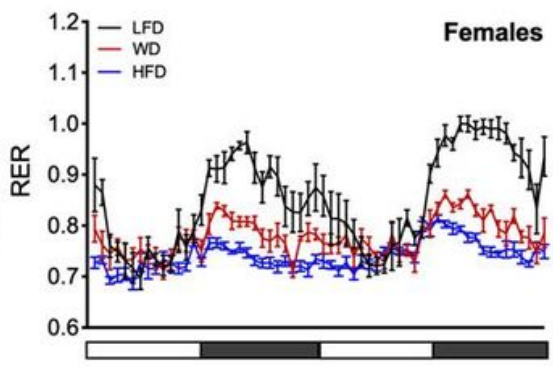

F

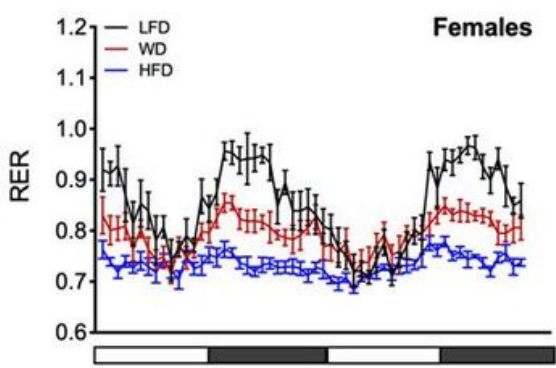

C

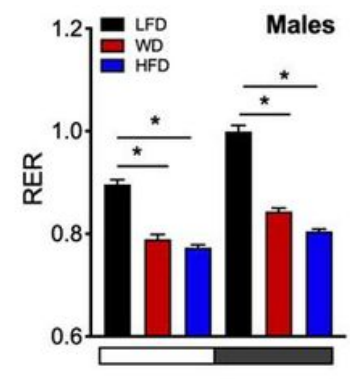

G

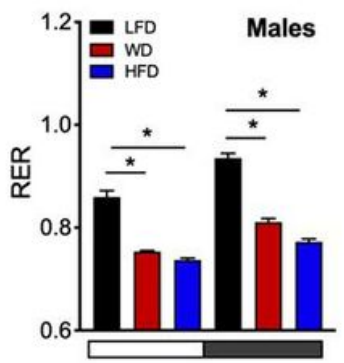

D
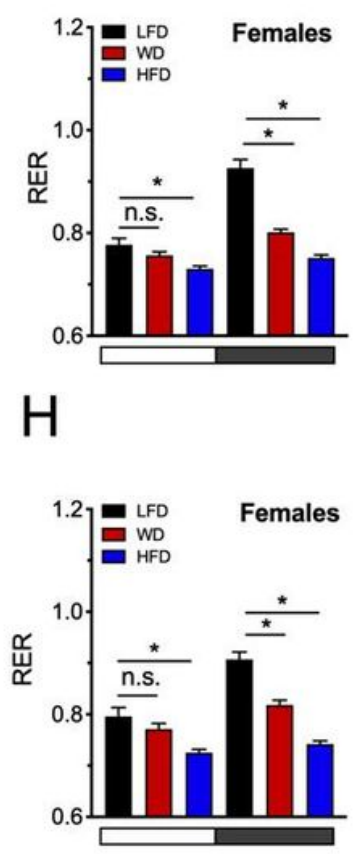

Figure 3

High fat dietary intervention results in a reduction in RER in both male and female mice. Male and female C57BL6/J mice were placed on a dietary intervention starting at 4 weeks of age and were singly housed in a PhenoMaster LabMaster indirect calorimeter. (A) Calculated RERs in male mice at 8 weeks of dietary intervention over a 48-hour period; (B) Calculated RERs in female mice at 8 weeks of dietary intervention over a 48-hour period. (C) Average RER values during the light or dark cycle in male mice; (D) Average RER values during the light or dark cycle in female mice. (E) RER values in male mice at 16 weeks of dietary intervention over a 48-hour period; (F) RER values in female mice at 16 weeks of dietary intervention over a 48-hour period; $(G)$ Average RER values during the light or dark cycle in male mice; (H) Average RER values during the light or dark cycle in female mice. Horizontal white x-axes bars denote light cycles $(0700-1900)$ and dark gray horizontal bars denote dark cycles (1900 - 0700). Results are displayed as means \pm SEM; $n=10$ per treatment group; Means were compared by one-way ANOVA followed by Tukey's posttest; *, $p<0.05$ for indicated comparisons. 
A

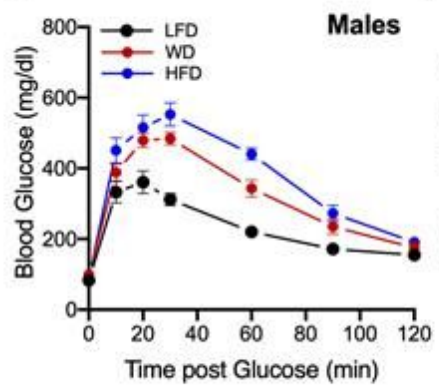

E
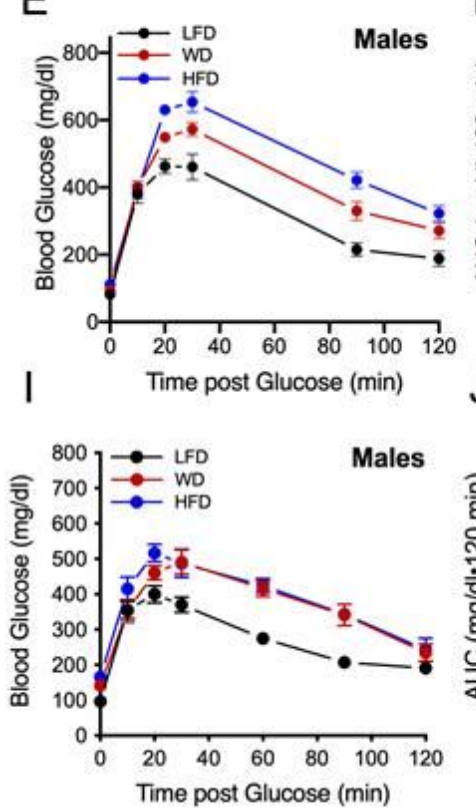

B

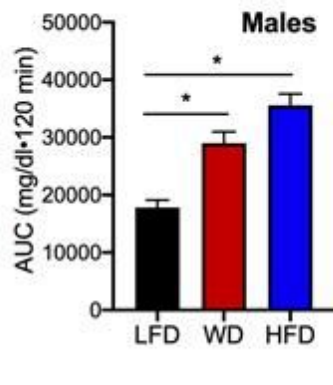

$\mathrm{F}$
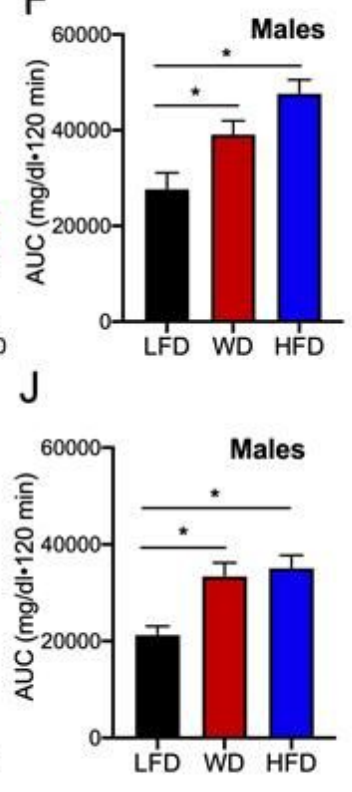

C
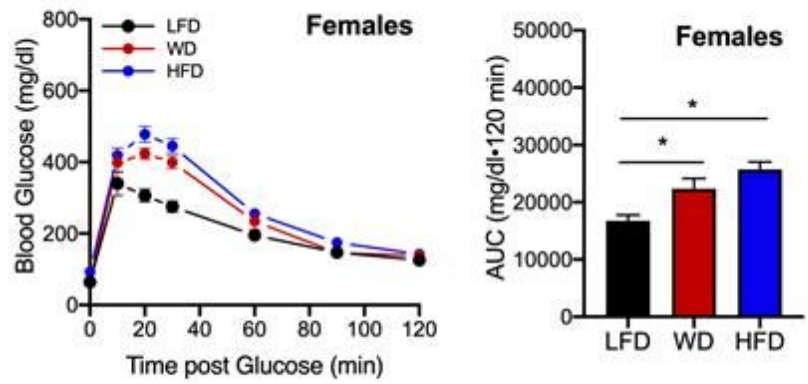

G

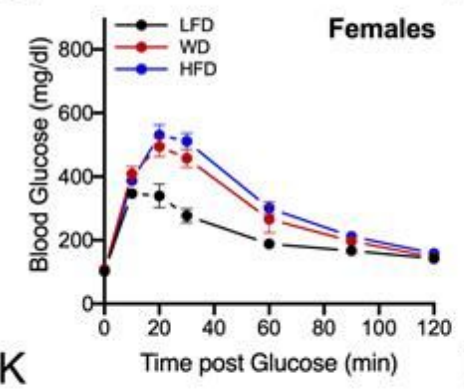

$\mathrm{H}$

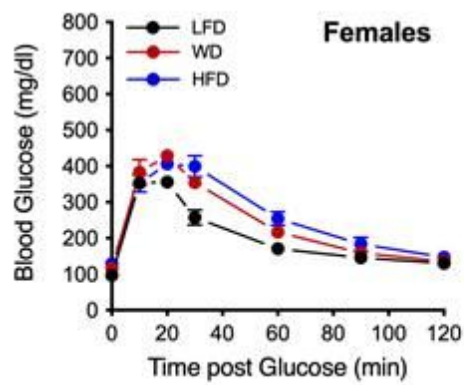

Figure 4

High dietary feeding worsens glucose tolerance in male C57BL6 mice compared to female mice. Male and female mice were placed on a dietary intervention starting at 4 weeks of age for 4,12 , or 20 weeks before a GTT. At the specified week of dietary intervention, mice were fasted overnight and injected intraperitoneally with $2 \mathrm{~g} / \mathrm{kg}$ of glucose. (A) GTT after $2 \mathrm{~g} / \mathrm{kg}$ glucose bolus in male mice at 4 weeks of dietary intervention; (B) Corresponding area under the curve from GTTs of male mice. (C) GTT of female mice at 4 weeks of dietary intervention; (D) Corresponding area under the curve from GTTs of female mice; (E) GTT of male mice at 12 weeks of dietary intervention; (F) Corresponding area under the curve from GTTs. (G) GTT of female mice at 12 weeks of dietary intervention; (H) Corresponding area under the curve from GTTs. (I) GTT of male mice at 20 weeks of dietary intervention; (J) corresponding area under the curve from GTTs; (K) GTT of female mice at 20 weeks of dietary intervention; (L) Corresponding area under the curve from GTTs. Results are displayed as means \pm SEM; $n=6-10$ per treatment group; Means were compared by one-way ANOVA followed by Tukey's posttest; *, $p<0.05$ for indicated comparisons.

A

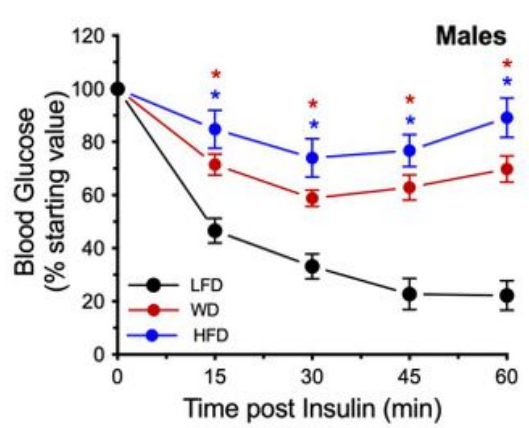

B

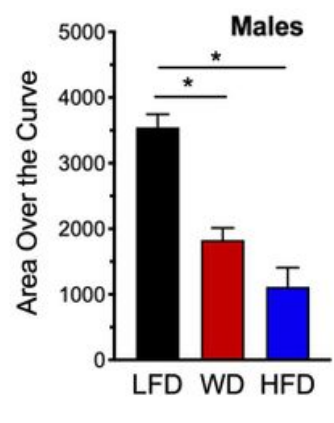

C

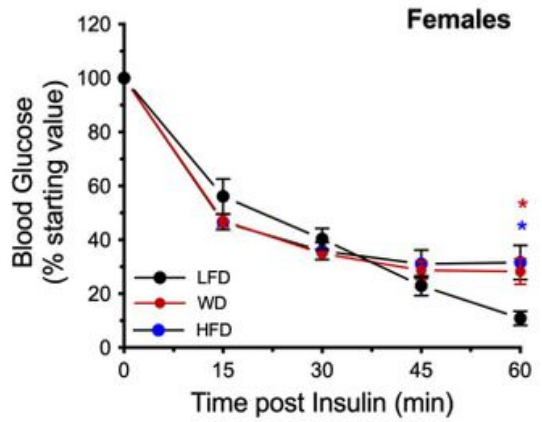

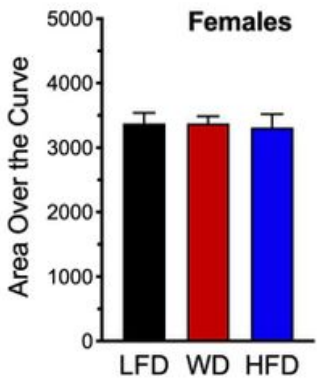




\section{Figure 5}

High fat feeding worsens insulin sensitivity in male mice. Male and female mice were placed on a dietary intervention starting at 4 weeks of age and subjected to an ITT at 22 weeks of diet. Mice were injected intraperitoneally with $0.75 \mathrm{U} / \mathrm{kg}$ insulin, and blood glucose concentrations were measured over the course of 60 minutes. (A) ITT in male mice and (B) corresponding area over the curve analysis of ITT performed in male mice; (C) ITT in female mice and (D) corresponding area over the curve analysis of ITT performed in female mice. Means were compared by one-way ANOVA followed by Tukey's posttest. Results are displayed as means \pm SEM; $n=5-8$ per treatment group; Means were compared by one-way ANOVA followed by Tukey's posttest; *, $\mathrm{p}<0.05$ for indicated comparisons.
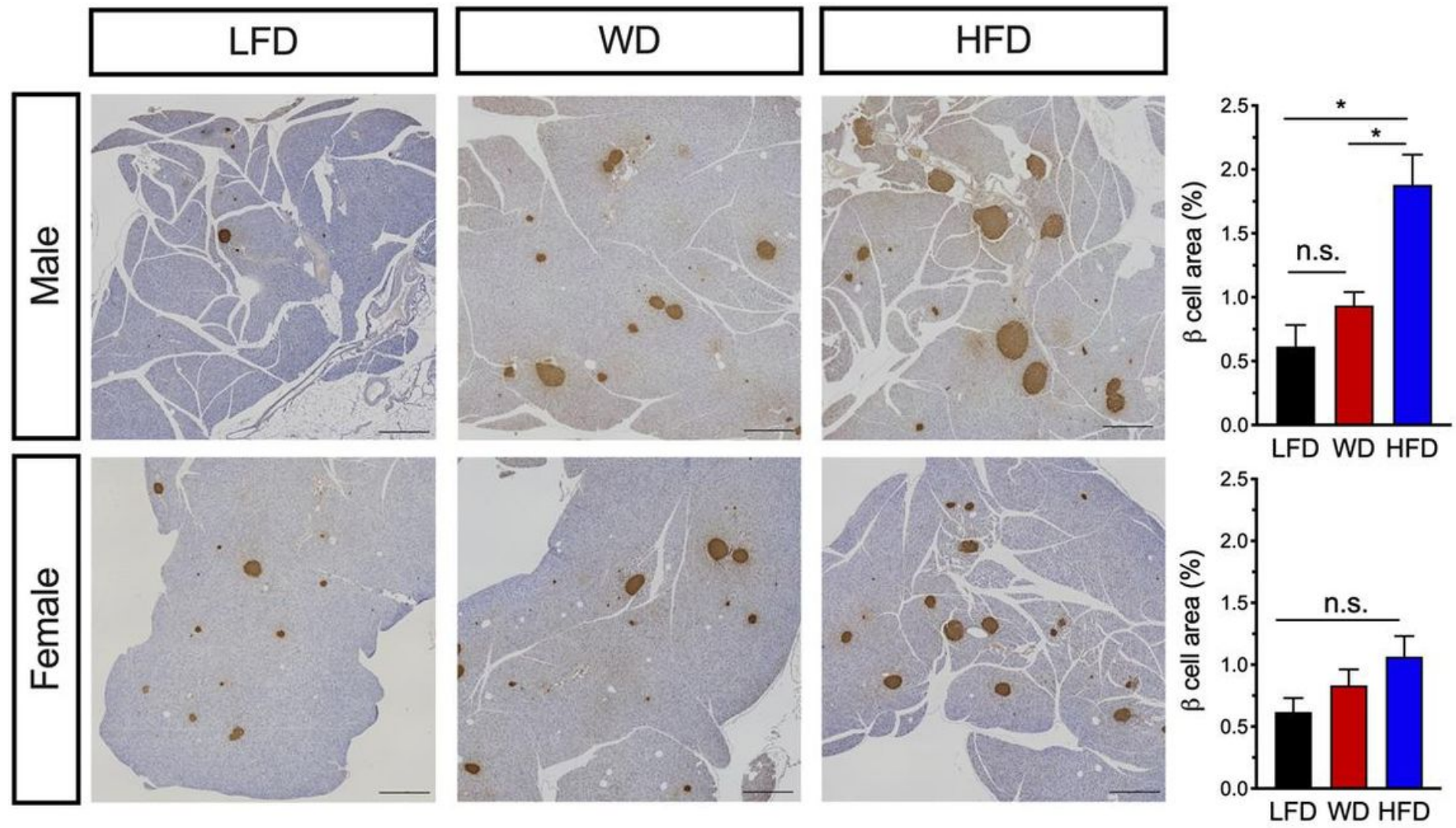

Figure 6

Male mice demonstrate increased $\beta$-cell area in response to either a WD or HFD. Male and female mice C57BL6/J mice were placed on a dietary intervention starting at 4 weeks of age. After 22 weeks of diet, mice were euthanized and pancreata harvested and stained for insulin. Left panel: Images of whole pancreatic sections from representative mice immunostained for insulin (brown) and counterstained with hematoxylin. Scale bars, $1000 \square \mathrm{m}$. Right panel: Quantitation of pancreatic $\beta$-cell area in male mice (top) and in female mice (bottom); Results are displayed as means \pm SEM; $n=3-5$ mice per treatment group. Means were compared by one-way ANOVA; *, $p<0.05$ for indicated comparisons. 